\title{
Connectivity Preservation in Nonholonomic Multi-Agent Systems: A Bounded Distributed Control Strategy
}

\author{
Amir Ajorlou and Amir G. Aghdam
}

\begin{abstract}
This paper is concerned with the connectivity preservation of a group of unicycles using a novel distributed control scheme. The proposed local controllers are bounded, and are capable of maintaining the connectivity of those pairs of agents which are initially within the connectivity range. Each local controller is designed in such a way that when an agent is about to lose connectivity with a neighbor, the lowest-order derivative of the agent's position that is neither zero nor perpendicular to the edge connecting the agent to the corresponding neighbor, makes an acute angle with this edge, which is shown to result in shrinking the edge. The proposed methodology is then used to develop bounded connectivity preserving control strategies for the consensus problem as one of the unprecedented contributions of this work. The theoretical results are validated by simulation.
\end{abstract}

\section{INTRODUCTION}

Network Connectivity is an important specification in the design of distributed control strategies for multi-agent systems. This problem has been extensively studied in the literature for different agent dynamics and various applications. In [1], [2], [3], potential functions and nonlinear weights for the edges of the interaction graph are used to generate local control signals for single-integrator agents, which tend to infinity when two neighboring agents are about to lose connectivity. Bounded distributed connectivity preserving control strategies for single-integrator agents are proposed in [4], [5].

In [5], a general class of controllers is introduced with the property that they preserve connectivity even if a subset of agents, namely static leaders, are to remain fixed. Connectivity of the graph of a network can also be preserved by controlling the second smallest eigenvalue of the corresponding Laplacian matrix, e.g. see [6], [7]. For double-integrator agents, [8] uses the same ideas as [1] to develop a hybrid control strategy which yields velocity alignment while maintaining connectivity and ensuring collision avoidance. The method also allows edge deletions using a distributed market-based control strategy. A cohesive overview of the main results of [6], [1], [3], [8] is presented in a unified framework in [9].

As for the unicycle agents, [10] proposes a discontinuous and time-invariant feedback control strategy to reach consensus in both positions and headings, while maintaining the connectivity of those neighbors which are initially in the connectivity range. However, the translational velocity of an agent may tend to infinity when it is about to lose connectivity with a neighbor. Thus, this technique may not be effective in practice since the actuators of the agents can only handle finite forces or torques.

This work has been supported by the Natural Sciences and Engineering Research Council of Canada (NSERC) under Grant RGPIN-262127-12.
A class of bounded distributed controllers for connectivity preservation of unicycles is presented as the unprecedented contribution of the present work, which is an extension of the results in [5] for single-integrator agents. The proposed control strategy maintains the connectivity of those agents that are initially in the connectivity range. Therefore, if the network is initially connected, it will remain connected at all times. Connectivity preservation is guaranteed even if some of the agents are to remain fixed. Smooth potential functions are used in order to obtain bounded control inputs. Although only the consensus application is discussed in this paper, a connectivity preserving containment controller can also be designed using an approach similar to [5].

\section{Problem Formulation}

Consider a set of $n$ nonholonomic agents in a 2D plane. Let $q_{i}$ and $\theta_{i}$ denote the position and heading of agent $i$, respectively $\left(i \in \mathbb{N}_{n}:=\{1, \ldots, n\}\right)$, where $q_{i}=\left[\begin{array}{ll}x_{i} & y_{i}\end{array}\right]^{T}$. The dynamics of each agent is given by

$$
\begin{aligned}
& \dot{x}_{i}=v_{i} \cos \theta_{i} \\
& \dot{y}_{i}=v_{i} \sin \theta_{i} \\
& \dot{\theta}_{i}=\omega_{i}
\end{aligned}
$$

where $v_{i}$ and $\omega_{i}$ are the translational and angular velocities of agent $i$, respectively. Each agent is assumed to be capable of measuring the relative positions and relative velocities of its neighbors (as defined later). Denote by $G=(V, E)$ the information flow graph, where $V=\{1, \ldots, n\}$ is the set of vertices and $E \subset V \times V$ is the set of edges. The information flow graph $G$ is assumed to be connected, undirected, and static (the case of dynamic information flow graphs is addressed later in Remark 1). Denote the set of neighbors of agent $i$ in $G$ by $N_{i}(G)$, and the degree of this agent by $d_{i}(G)$. Two agents $i$ and $j$ are said to be in the connectivity range if $\left\|q_{i}-q_{j}\right\|<d$, for a prespecified positive real number $d$, where $\|\cdot\|$ denotes the Euclidean norm. It is assumed that all agents in $N_{i}(G)$ are initially located in the connectivity range of agent $i$, for all $i \in \mathbb{N}_{n}$. It is also assumed that each agent belongs to either the set of leaders $\mathscr{L}$ or the set of followers $\mathscr{F}$, and that the leaders are static, i.e. $v_{i} \equiv 0, \omega_{i} \equiv 0$ for all $i \in \mathscr{L}$. The main objective is to design a distributed controller for the followers to preserve connectivity. More precisely, it is desired to find a control scheme for the followers such that if the inequality $\left\|q_{i}(t)-q_{j}(t)\right\|<d$ holds for all $(i, j) \in E$ at $t=0$, then it holds at any $t>0$ as well. The proposed control scheme is then used to develop connectivity preserving control strategies for the consensus problem.

Definition 1: For every agent $i$, the following functions are 
introduced

$$
\begin{aligned}
\sigma_{i}(t) & :=\frac{1}{2} \sum_{j \in N_{i}(G)}\left\|q_{i}(t)-q_{j}(t)\right\|^{2} \\
\pi_{i}(t) & :=\frac{1}{2} \prod_{j \in N_{i}(G)}\left(d^{2}-\left\|q_{i}(t)-q_{j}(t)\right\|^{2}\right) \\
\pi_{i j}(t) & :=\prod_{\substack{k \in N_{i}(G) \\
k \neq j}}\left(d^{2}-\left\|q_{i}(t)-q_{k}(t)\right\|^{2}\right)
\end{aligned}
$$

Definition 2: The index of a real or vector-valued function $f$ at time $t$, denoted by $\rho(f(t))$, is defined as the smallest natural number $n$ for which $f^{(n)}(t) \neq 0$, where $f^{(n)}(t)$ is the $n$-th derivative of $f$ at time $t$.

\section{Connectivity Preserving Controller Design}

Similar to [5], consider a set of distributed smooth potential functions of the form $h_{i}\left(\sigma_{i}, \pi_{i}\right), i \in \mathscr{F}$, with the following properties for all $\sigma_{i} \in \mathbb{R}^{+}$

$$
\begin{aligned}
& \frac{\partial h_{i}}{\partial \sigma_{i}}\left(\sigma_{i}, 0\right)=0 \\
& \frac{\partial h_{i}}{\partial \pi_{i}}\left(\sigma_{i}, 0\right)<0
\end{aligned}
$$

Note that under the conditions given by (2), when agent $i$ is about to lose connectivity (i.e. $\pi_{i}=0$ ), any change in $h_{i}$ results only from a change in $\pi_{i}$, and if the agents move in a direction that leads to a decrease in $h_{i}$, then the connectivity will improve (i.e., $\pi_{i}$ will increase). Define $r_{i}=-\frac{\partial h_{i}}{\partial q_{i}}$, and denote by $\theta_{i}^{*}$ the angle of $r_{i}=\left[\begin{array}{ll}r_{i x} & r_{i y}\end{array}\right]^{T}$. For every agent $i \in \mathscr{F}$, consider a controller of the form

$$
\begin{aligned}
v_{i} & =\left\|r_{i}\right\| \cos \left(\theta_{i}-\theta_{i}^{*}\right) \\
\omega_{i} & =\dot{\theta}_{i}^{*}-\left(\theta_{i}-\theta_{i}^{*}\right)
\end{aligned}
$$

Calculation of $r_{i}$ and $\theta_{i}^{*}$ requires only the relative positions of the neighbors of agent $i$. It is straightforward to show that calculating $\dot{\theta}_{i}^{*}$ also requires the relative velocities of the neighbors of agent $i$.

Define $\mathscr{T}=\left\{t \mid \exists(i, j) \in E:\left\|q_{i}(t)-q_{j}(t)\right\| \geq d\right\}$. In order to prove that the controller given by (3) is connectivity preserving, it suffices to show that $\mathscr{T}=\emptyset$. Assume $\mathscr{T} \neq \emptyset$, and let $t_{0}=\inf _{t \in \mathscr{T}} t$. This implies that $\left\|q_{i}(t)-q_{j}(t)\right\| \leq d$, for all $(i, j) \in E$ and $t \leq t_{0}$, where the equality holds for at least one edge at $t=t_{0}$. Construct a graph $G_{d}=\left(V_{d}, E_{d}\right)$ as the union of the edges $(i, j) \in E$ for which $\left\|q_{i}\left(t_{0}\right)-q_{j}\left(t_{0}\right)\right\|=d$, i.e. the edges that are at the critical distance at $t=t_{0}$. Define

$$
s_{i j}(t)=\left\|q_{i}(t)-q_{j}(t)\right\|^{2}, \quad \forall(i, j) \in E_{d}
$$

Now, assume that $s_{i j}$ is decreasing for some $(i, j) \in E_{d}$, in an open interval $\left(t_{a}, t_{b}\right)$, where $t_{a}<t_{0}<t_{b}$. For such an edge and for every $t_{a} \leq t<t_{0}$, the inequality $\left\|q_{i}(t)-q_{j}(t)\right\|>$ $\left\|q_{i}\left(t_{0}\right)-q_{j}\left(t_{0}\right)\right\|=d$ holds which is in contradiction with the fact that $\left\|q_{i}(t)-q_{j}(t)\right\| \leq d$, for all $(i, j) \in E$ and $t \leq t_{0}$. This rejects the assumption that $\mathscr{T} \neq \emptyset$, and hence the control law given by (3) is connectivity preserving. Thus, in order to prove the connectivity preservation for the proposed controller, it suffices to show that the edge described above exists. In the sequel, some important properties of the graph $G_{d}$ are presented, which will be used later in Theorem 1 for finding such an edge.

Define the rotation matrix

$$
\operatorname{Rot}(\alpha)=\left[\begin{array}{cc}
\cos \alpha & -\sin \alpha \\
\sin \alpha & \cos \alpha
\end{array}\right]
$$

where $\alpha$ is the rotation angle in radians. It is straightforward to verify that $\frac{d}{d t} \operatorname{Rot}(\alpha)=\dot{\alpha} \operatorname{Rot}\left(\alpha+\frac{\pi}{2}\right)$. Consider an agent $i \in \mathscr{F}$; from (1a), (1b), (3a), and on noting that $r_{i}=\left\|r_{i}\right\|\left[\cos \theta_{i}^{*} \sin \theta_{i}^{*}\right]^{T}$, one can obtain

$$
\begin{aligned}
\dot{q}_{i} & =v_{i}\left[\begin{array}{c}
\cos \theta_{i} \\
\sin \theta_{i}
\end{array}\right]=\operatorname{Rot}\left(\theta_{i}-\theta_{i}^{*}\right) r_{i} \cos \left(\theta_{i}-\theta_{i}^{*}\right) \\
& =\frac{1}{2}\left[\begin{array}{cc}
1+\cos 2\left(\theta_{i}-\theta_{i}^{*}\right) & -\sin 2\left(\theta_{i}-\theta_{i}^{*}\right) \\
\sin 2\left(\theta_{i}-\theta_{i}^{*}\right) & 1+\cos 2\left(\theta_{i}-\theta_{i}^{*}\right)
\end{array}\right] r_{i} \\
& =\frac{1}{2}\left(\operatorname{Rot}\left(2 \alpha_{i}\right)+I_{2}\right) r_{i}
\end{aligned}
$$

where $\alpha_{i}=\theta_{i}-\theta_{i}^{*}$, and $I_{2}$ is the $2 \times 2$ identity matrix. It results from (3b) that $\dot{\alpha}_{i}=-\alpha_{i}$. Furthermore, since $\frac{\partial h_{i}}{\partial q_{i}}=\frac{\partial h_{i}}{\partial \sigma_{i}} \frac{\partial \sigma_{i}}{\partial q_{i}}+$ $\frac{\partial h_{i}}{\partial \pi_{i}} \frac{\partial \pi_{i}}{\partial q_{i}}$, one can rewrite $r_{i}$ as

$$
r_{i}=-\sum_{j \in N_{i}(G)}\left(\frac{\partial h_{i}}{\partial \sigma_{i}}-\frac{\partial h_{i}}{\partial \pi_{i}} \pi_{i j}\right)\left(q_{i}-q_{j}\right)
$$

The next lemma shows that $G_{d}$ is a union of trees, with at least one follower leaf.

Lemma 1: The graph $G_{d}$ is acyclic, and there exists at least one leaf in $G_{d}$ which is a follower.

Proof. Suppose that $G_{d}$ contains a cycle $\mathscr{C}$. Let $y(t)$ denote the positions of the agents belonging to this cycle, and $x(t)$ denote the positions of the rest of the agents along with the headings of all agents. If $y(t)=y\left(t_{0}\right)$ for some $t \geq 0$, one can easily show that $\pi_{i j}(t)=0$ for any $i \in \mathscr{F}$ on $\mathscr{C}$ and $j \in$ $N_{i}(G)$. This is due to the fact that every follower $i$ on $\mathscr{C}$ is at distance $d$ from its two neighbors on this cycle. Denote these two neighbors by $i_{1}$ and $i_{2}$; then, at least one of the two terms $\left(d^{2}-\left\|q_{i}-q_{i_{1}}\right\|^{2}\right)$ and $\left(d^{2}-\left\|q_{i}-q_{i_{2}}\right\|^{2}\right)$ appear in $\pi_{i j}$, making it zero. Now, it follows from (5) that $r_{i}(t)=0$ and hence $v_{i}(t)=$ 0 for any $i \in \mathscr{F}$ on $\mathscr{C}$. Using this argument, it is easy to show that $x$ and $y$ satisfy the conditions of Lemma 7 in [5], and hence $y(t)=y\left(t_{0}\right)$ for all $t \geq 0$. In particular $y(0)=y\left(t_{0}\right)$, implying that the initial distance between some agents is equal to $d$, which contradicts the assumption that $\left\|q_{i}(0)-q_{j}(0)\right\|<d$ for all $(i, j) \in E$. This proves that $G_{d}$ is acyclic.

Now, let $\mathscr{P}$ be the longest path in $G_{d}$ and denote by $u$ and $v$ the vertices at the two ends of this path. Clearly, $d_{u}\left(G_{d}\right)=$ $d_{v}\left(G_{d}\right)=1$, which means $u$ and $v$ are two leafs of $G_{d}$. Assume that both $u$ and $v$ are static leaders. Then, every agent $i \in \mathscr{F}$ on $\mathscr{P}$ has two neighbors on this path located at distance $d$ from it. Therefore, an argument similar to the one given above results that the agents on this path have been fixed from the beginning, which again contradicts the assumption that $\left\|q_{i}(0)-q_{j}(0)\right\|<$ $d$, for all $(i, j) \in E$. Thus, at least one of the two leafs $u$ and $v$ is a follower.

The next 3 lemmas will be used later in Theorem 1 to find the derivative of $s_{i j}$ for an edge connected to a follower leaf.

Lemma 2: Consider an agent $i \in \mathscr{F}$ in $G_{d}$ with $d_{i}\left(G_{d}\right)=1$, and let agent $j$ be the one for which $\left\|q_{i}-q_{j}\right\|=d$. If $\alpha_{i} \neq \pm \frac{\pi}{2}$, 
then $\left(q_{i}-q_{j}\right)^{T} \dot{q}_{i}<0$.

Proof. It is straightforward to show that for agent $i$ described in the lemma the relation $r_{i}=\frac{\partial h_{i}}{\partial \pi_{i}} \pi_{i j}\left(q_{i}-q_{j}\right)$ holds. Therefore,

$$
\left(q_{i}-q_{j}\right)^{T} \dot{q}_{i}=\frac{r_{i}^{T}}{2 \frac{\partial h_{i}}{\partial \pi_{i}} \pi_{i j}}\left(\operatorname{Rot}\left(2 \alpha_{i}\right)+I_{2}\right) r_{i}=\frac{\left(1+\cos 2 \alpha_{i}\right)}{2 \frac{\partial h_{i}}{\partial \pi_{i}} \pi_{i j}}\left\|r_{i}\right\|^{2}
$$

The proof follows on noting that $1+\cos 2 \alpha_{i}>0$ for any $\alpha_{i} \neq$ $\pm \frac{\pi}{2}$, and that $\frac{\partial h_{i}}{\partial \pi_{i}}<0$ (from (2b)).

Lemma 3: Consider an agent $i \in \mathscr{F}$ in $G_{d}$ with $d_{i}\left(G_{d}\right)=1$, and let agent $j$ be the one for which $\left\|q_{i}-q_{j}\right\|=d$. If $\dot{q}_{i}=$ $\dot{q}_{j}=0$, then $\dot{r}_{i}=\frac{d}{d t}\left(\frac{\partial h_{i}}{\partial \pi_{i}} \pi_{i j}\right)\left(q_{i}-q_{j}\right)$.

Proof. Since $d^{2}-\left\|q_{i}-q_{j}\right\|^{2}=0$, for any $l \in N_{i}-\{j\}$ :

$$
\begin{aligned}
\dot{\pi}_{i l} & =\frac{d}{d t}\left(d^{2}-\left\|q_{i}-q_{j}\right\|^{2}\right) \prod_{\substack{k \in N_{i}(G) \\
k \neq l, j}}\left(d^{2}-\left\|q_{i}(t)-q_{k}(t)\right\|^{2}\right) \\
& =-2\left(q_{i}-q_{j}\right)^{T}\left(\dot{q}_{i}-\dot{q}_{j}\right) \prod_{\substack{k \in N_{i}(G) \\
k \neq l, j}}\left(d^{2}-\left\|q_{i}(t)-q_{k}(t)\right\|^{2}\right) \\
& =0
\end{aligned}
$$

This also implies that $\dot{\pi}_{i}=0$. On the other hand,

$$
\frac{d}{d t}\left(\frac{\partial h_{i}}{\partial \sigma_{i}}\right)=\frac{\partial^{2} h_{i}}{\partial \sigma_{i}^{2}} \dot{\sigma}_{i}+\frac{\partial^{2} h_{i}}{\partial \pi_{i} \partial \sigma_{i}} \dot{\pi}_{i}
$$

From (2a), it is straightforward to show that $\frac{\partial^{2} h_{i}}{\partial \sigma_{i}^{2}}=0$. Since $\dot{\pi}_{i}$ is also zero (as noted above), hence $\frac{d}{d t}\left(\frac{\partial h_{i}}{\partial \sigma_{i}}\right)=0$. The proof follows now on noting that

$$
\begin{aligned}
\dot{r}_{i}= & -\sum_{l \in N_{i}(G)}\left(\frac{\partial h_{i}}{\partial \sigma_{i}}-\frac{\partial h_{i}}{\partial \pi_{i}} \pi_{i l}\right)\left(\dot{q}_{i}-\dot{q}_{l}\right) \\
& -\sum_{l \in N_{i}(G)} \frac{d}{d t}\left(\frac{\partial h_{i}}{\partial \sigma_{i}}-\frac{\partial h_{i}}{\partial \pi_{i}} \pi_{i l}\right)\left(q_{i}-q_{l}\right)
\end{aligned}
$$

Lemma 4: Consider an agent $i \in \mathscr{F}$ in $G_{d}$ with $d_{i}\left(G_{d}\right)=1$, and let agent $j$ be the one for which $\left\|q_{i}-q_{j}\right\|=d$. Also, assume $\alpha_{i}= \pm \frac{\pi}{2}$. The following results hold:

a) $\left(q_{i}-q_{j}\right)^{T} \ddot{q}_{i}=0$

b) If $\dot{q}_{j}=0$, then $\left(q_{i}-q_{j}\right)^{T} q_{i}^{(3)}<0$

Proof.

Part (a). Since $\operatorname{Rot}\left(2 \alpha_{i}\right)=\operatorname{Rot}( \pm \pi)=-I_{2}$, thus,

$\ddot{q}_{i}=\frac{1}{2}\left(\operatorname{Rot}\left(2 \alpha_{i}\right)+I_{2}\right) \dot{r}_{i}+\dot{\alpha}_{i} \operatorname{Rot}\left(2 \alpha_{i}+\frac{\pi}{2}\right) r_{i}=-\alpha_{i} \operatorname{Rot}\left(-\frac{\pi}{2}\right) r_{i}$

As a result,

$$
\left(q_{i}-q_{j}\right)^{T} \ddot{q}_{i}=-\frac{\alpha_{i}}{\frac{\partial h_{i}}{\partial \pi_{i}} \pi_{i j}} r_{i}^{T} \operatorname{Rot}\left(-\frac{\pi}{2}\right) r_{i}=0
$$

Part (b). One can easily find the third derivative of $q_{i}$ as

$$
q_{i}^{(3)}=\left(\alpha_{i} \operatorname{Rot}\left(-\frac{\pi}{2}\right)+2 \alpha_{i}^{2} I_{2}\right) r_{i}-2 \alpha_{i} \operatorname{Rot}\left(-\frac{\pi}{2}\right) \dot{r}_{i}
$$

If $\dot{q}_{j}=0$, then Lemma 3 yields

$$
\begin{aligned}
\left(q_{i}-q_{j}\right)^{T} \operatorname{Rot}\left(-\frac{\pi}{2}\right) \dot{r}_{i} & =\frac{d}{d t}\left(\frac{\partial h_{i}}{\partial \pi_{i}} \pi_{i j}\right)\left(q_{i}-q_{j}\right)^{T} \operatorname{Rot}\left(-\frac{\pi}{2}\right)\left(q_{i}-q_{j}\right) \\
& =0
\end{aligned}
$$

Therefore,

$$
\begin{aligned}
\left(q_{i}-q_{j}\right)^{T} q_{i}^{(3)} & =\frac{r_{i}^{T}}{\frac{\partial h_{i}}{\partial \pi_{i}} \pi_{i j}}\left(\alpha_{i} \operatorname{Rot}\left(-\frac{\pi}{2}\right)+2 \alpha_{i}^{2} I_{2}\right) r_{i} \\
& =\frac{2 \alpha_{i}^{2}}{\frac{\partial h_{i}}{\partial \pi_{i}} \pi_{i j}}\left\|r_{i}\right\|^{2} \\
& <0
\end{aligned}
$$

It follows from Lemma 2 that under the proposed control law, when a follower leaf $i$ in $G_{d}$ is about to lose connectivity with a neighbor, it is forced to move in such a way that it makes an acute angle with respect to the corresponding edge. If, however, the heading of the agent is perpendicular to this edge, then, according to Lemma 4 , the velocity of the agent is zero and the acceleration of the agent is perpendicular to this edge. The derivative of the acceleration in this case makes an acute angle with this edge, aiming to shrink it.

Another outcome of the above lemmas is that for a follower leaf $i$ in $G_{d}$, if the heading of the agent is perpendicular to $r_{i}$, then $\rho\left(q_{i}\right)=2$; otherwise, $\rho\left(q_{i}\right)=1$. Also, note that since the leaders are static, the index $\rho\left(q_{i}\right)$ is $\infty$ for every $i \in \mathscr{L}$. The following three lemmas will be used in Theorem 1 to find the derivatives of $s_{i j}$ for an edge connected to a leaf whose other end is a non-leaf follower.

Lemma 5: Consider a follower agent $i$ in $G_{d}$, and define $\eta=\min _{j \in N_{i}(G)}\left\{\rho\left(\pi_{i j}\right)\right\}$. If $d_{i}\left(G_{d}\right) \geq 2$, then $\rho\left(q_{i}\right) \geq \eta+1$.

Proof. The proof is similar to that of Lemma 3 in [5], using the relation $\rho\left(q_{i}\right) \geq \rho\left(r_{i}\right)+1$.

Lemma 6: Consider a follower agent $i$ in $G_{d}$, and let $v$ be one of the (possibly multiple) neighbors of this agent in $G_{d}$ for which $\rho\left(q_{v}\right)=\max _{j \in N_{i}\left(G_{d}\right)}\left\{\rho\left(q_{j}\right)\right\}$. Then

$$
\rho\left(q_{i}\right) \geq 1+\sum_{\substack{j \in N_{i}\left(G_{d}\right) \\ j \neq v}} \rho\left(q_{j}\right)
$$

Proof. The proof is similar to that of Lemma 4 in [5], and is omitted here.

Lemma 7: Consider an agent $i \in \mathscr{F}$ in $G_{d}$ with $d_{i}\left(G_{d}\right) \geq 2$. If $\rho\left(q_{j}\right)=2$ for any agent $j \in V_{d} \cap \mathscr{F}$ with $d_{j}\left(G_{d}\right)=1$, then $\rho\left(q_{i}\right) \geq 4$.

Proof. If $d_{i}\left(G_{d}\right) \geq 3$, then Lemma 6 yields $\rho\left(q_{i}\right) \geq 1+2+$ $2=5$, and hence the statement of the present lemma holds in this case. Now, for the case when $d_{i}\left(G_{d}\right)=2$, Lemma 6 implies that $\rho\left(q_{i}\right) \geq 1+2=3$. Let $N_{i}\left(G_{d}\right)=\{j, k\}$. Using the equality $\pi_{i j}=d^{2}-\left\|q_{i}-q_{k}\right\|^{2}$, it can be easily shown that $\dot{\pi}_{i j}=0$ and $\ddot{\pi}_{i j}=\left(q_{i}-q_{k}\right)^{T} \ddot{q}_{k}$. It is now aimed to prove that $\ddot{\pi}_{i j}=0$. If $k \in \mathscr{L}$, then $\rho\left(q_{k}\right)=\infty$. Also, if $k \in \mathscr{F}$ and $d_{k}\left(G_{d}\right) \geq 2$, then $\rho\left(q_{k}\right) \geq 3$. Hence, in these two cases $\ddot{q}_{k}=0$, and subsequently $\ddot{\pi}_{i j}=0$. On the other hand, if $k \in \mathscr{F}$ and $d_{k}\left(G_{d}\right)=1$, then from the assumption of the lemma $\rho\left(q_{k}\right)$ equals 2 , implying that $\alpha_{k}= \pm \frac{\pi}{2}$. Thus, Lemma 4 yields $\left(q_{i}-q_{k}\right)^{T} \ddot{q}_{k}=0$. It follows from the above argument that $\dot{\pi}_{i j}=\ddot{\pi}_{i j}=0$. Similarly, $\dot{\pi}_{i k}=$ $\ddot{\pi}_{i k}=0$. Therefore, $\eta=\min \left\{\rho\left(\pi_{i j}\right), \rho\left(\pi_{i k}\right)\right\} \geq 3$, and hence it is concluded from Lemma 5 that $\rho\left(q_{i}\right) \geq 4$.

Theorem 1: Consider a set of $n$ nonholonomic agents in a plane with dynamics of the form (1), and assume that the leaders are static. Assume also that $n$ potential functions are 
given which satisfy the conditions in (2). Then, the distributed controller (3) for the followers is connectivity preserving.

Proof. As stated earlier, to prove this theorem it suffices to show that for some $(i, j) \in E_{d}$ the function $s_{i j}$ defined by (4) is decreasing in an open interval around $t_{0}$. It is shown in the sequel that any edge connected to a follower leaf of index one is an appropriate candidate (for the case where the index of every follower leaf is 2 , any edge connected to any follower leaf can be selected). Two cases are considered here:

i) $G_{d}$ has at least one follower leaf of index 1 . Let $i$ be the vertex representing one of such followers, and $j$ be the vertex for which $\left\|q_{i}-q_{j}\right\|=d$. Then, $\dot{s}_{i j}=2\left(q_{i}-q_{j}\right)^{T} \dot{q}_{i}+2\left(q_{j}-\right.$ $\left.q_{i}\right)^{T} \dot{q}_{j}$. Since $\rho\left(q_{i}\right)=1$, thus $\alpha_{i} \neq \pm \frac{\pi}{2}$, and Lemma 2 implies that $\left(q_{i}-q_{j}\right)^{T} \dot{q}_{i}<0$. If $\rho\left(q_{j}\right) \geq 2$, then $\dot{q}_{j}=0$, and hence $\dot{s}_{i j}<0$. If $\rho\left(q_{j}\right)=1$, then $j$ is also a follower leaf of $G_{d}$, and similarly $\left(q_{j}-q_{i}\right)^{T} \dot{q}_{j}<0$, which yields $\dot{s}_{i j}<0$. Therefore, $s_{i j}$ is decreasing in an open interval around $t_{0}$, which completes the proof for this case.

ii) The index of every follower leaf in $G_{d}$ is 2. Consider a follower leaf $i$ in $G_{d}$, and let $j$ be the vertex for which $\| q_{i}-$ $q_{j} \|=d$. Clearly, $\dot{s}_{i j}=0$ and also $\ddot{s}_{i j}=2\left(q_{i}-q_{j}\right)^{T} \ddot{q}_{i}+2\left(q_{j}-\right.$ $\left.q_{i}\right)^{T} \ddot{q}_{j}$. Lemma 4 implies that $\left(q_{i}-q_{j}\right)^{T} \ddot{q}_{i}=0$. Similarly, if $j$ belongs to $\mathscr{F}$ and is a leaf, then $\left(q_{j}-q_{i}\right)^{T} \ddot{q}_{j}=0$. If, on the other hand, $j$ is a static leader or is a follower but not a leaf, then $\ddot{q}_{j}=0$. Therefore, regardless of $j$ being a leaf or not, the equality $\ddot{s}_{i j}=0$ holds. To find the third derivative of $s_{i j}$, note that since the index of every follower in $G_{d}$ is assumed to be $2, s_{i j}^{(3)}=2\left(q_{i}-q_{j}\right)^{T} q_{i}^{(3)}+2\left(q_{j}-q_{i}\right)^{T} q_{j}^{(3)}$. From Lemma 4 , $\left(q_{i}-q_{j}\right)^{T} q_{i}^{(3)}<0$. If $j$ belongs to $\mathscr{F}$ and is a leaf, then it can be concluded in a similar way that $\left(q_{j}-q_{i}\right)^{T} q_{j}^{(3)}<0$, which along with the above inequality yields $s_{i j}^{(3)}<0$. If $j \in \mathscr{F}$ and $d_{j}\left(G_{d}\right) \geq 2$, then Lemma 7 implies that $\rho\left(q_{j}\right) \geq 4$ and hence $q_{j}^{(3)}=0$, resulting in $s_{i j}^{(3)}<0$. The same result holds also if $j$ is a static leader. Now, it is deduced from $\dot{s}_{i j}=\ddot{s}_{i j}=0$ and $s_{i j}^{(3)}<0$ that $s_{i j}$ is decreasing in an open interval around $t_{0}$, which completes the proof.

Remark 1: The connectivity preservation results presented so far can be easily extended to the case of dynamic edge addition, where new edges may be added to the information flow graph once two agents enter the connectivity range. Suppose that new edges are added to the information flow graph at the time instants $t_{1}, t_{2}, \ldots$, and denote by $G\left(t_{k}\right)$ the resultant information flow graph at $t=t_{k}, k=1,2, \ldots$. For any edge $e \in E\left(t_{k}\right)$, the corresponding agents remain in the connectivity range during the time interval $\left[t_{k}, t_{k+1}\right]$ according to Theorem 1. This, along with the fact that $E \subseteq E\left(t_{1}\right) \subseteq$ $E\left(t_{2}\right) \subseteq \ldots$, implies that for any edge of the information flow graph the corresponding agents remain in the connectivity range at all times once the edge is created.

\section{A Bounded CONNECTIVITY PRESERVING CONSENSUS AlgorithM FOR UNICYCLES}

Consider a team of $n$ unicycles in a 2D plane with the dynamics of the form (1), and assume that the corresponding information flow graph $G$ is static and is a tree. Assume also that $n$ potential functions are given which are analytic and, in

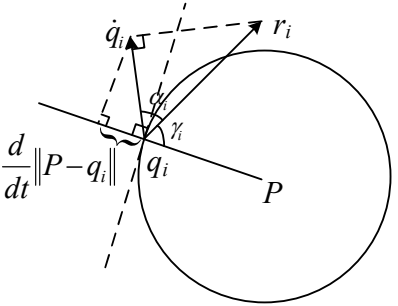

Fig. 1: The configuration described in Lemma 8 for the case when $\left|\alpha_{i}+\gamma_{i}\right|>\frac{\pi}{2}$.

addition to (2), satisfy the following constraints

$$
\frac{\partial h_{i}}{\partial \sigma_{i}}\left(\sigma_{i}, \pi_{i}\right)>0, \frac{\partial h_{i}}{\partial \pi_{i}}\left(\sigma_{i}, \pi_{i}\right) \leq 0, \quad \text { for } \sigma_{i} \geq 0 \text { and } \pi_{i}>0
$$

Then, it is desired to show that using the controller given by (3), the agents converge to consensus while preserving connectivity. Connectivity preservation follows directly from condition (2) and the results of the previous section. To prove the convergence to consensus, a few lemmas and theorems are presented in the sequel.

It follows from connectivity preservation that $\sigma_{i}$ and $\pi_{i}$ in (5) are bounded, and so are $\frac{\partial h_{i}}{\partial \sigma_{i}}$ and $\frac{\partial h_{i}}{\partial \pi_{i}}$ as analytic functions of $\sigma_{i}$ and $\pi_{i}$. Thus, there exists a positive real number $r_{M}$ such that $\left\|r_{i}(t)\right\| \leq r_{M}$, for all $t \geq 0$ and all $i \in \mathbb{N}_{n}$. For a fixed point $P \in \mathbb{R}^{2}$, define $R^{P}(t)=\max _{i \in \mathbb{N}_{n}}\left\|P-q_{i}(t)\right\|$. Denote by $\frac{d^{+}}{d t} R^{P}(t)$ the right derivative of $R^{P}(t)$ with respect to $t$. The next lemma will be used to find an upper bound for $\frac{d^{+}}{d t} R^{P}(t)$.

Lemma 8: Let $i$ be an agent for which $\left\|P-q_{i}(t)\right\|=R^{P}(t)$ (i.e., the farthest agent from $P$ at time $t$ ). Also, assume $\left|\alpha_{i}(t)\right| \leq \frac{\pi}{2}$. Then $\frac{d}{d t}\left\|P-q_{i}(t)\right\| \leq r_{M}\left|\alpha_{i}(t)\right|$.

Proof. Denote by $\gamma_{i}$ the angle between $P-q_{i}$ and $r_{i}$. Also, define $\beta_{i j}=\frac{\partial h_{i}}{\partial \sigma_{i}}-\frac{\partial h_{i}}{\partial \pi_{i}} \pi_{i j}$, and write (5) as $r_{i}=$ $-\sum_{j \in N_{i}(G)} \beta_{i j}\left(q_{i}-q_{j}\right)$. It follows from (6) and connectivity preservation that $\beta_{i j}>0$. Moreover, since agent $i$ is the farthest agent from $P$, the circle centered at $P$ with the radius $\left\|P-q_{i}\right\|$ contains all agents, and hence $\left(P-q_{i}\right)^{T}\left(q_{j}-q_{i}\right) \geq 0$ for all $j \in \mathbb{N}_{n}$. Therefore $\left(P-q_{i}\right)^{T} r_{i} \geq 0$, which implies that $\left|\gamma_{i}\right| \leq \frac{\pi}{2}$. On the other hand, it is straightforward to show that

$$
\frac{d}{d t}\left\|P-q_{i}(t)\right\|=-\frac{\left(P-q_{i}\right)^{T}}{\left\|P-q_{i}\right\|} \dot{q}_{i}=\left\|r_{i}\right\| \sin \left(\left|\alpha_{i}+\gamma_{i}\right|-\frac{\pi}{2}\right) \cos \alpha_{i}
$$

(this is illustrated in Fig. 1 for the case where $\left|\alpha_{i}+\gamma_{i}\right|>\frac{\pi}{2}$ ). It follows from $\left|\alpha_{i}\right| \leq \frac{\pi}{2}$ and $\left|\gamma_{i}\right| \leq \frac{\pi}{2}$ that $-\frac{\pi}{2} \leq\left|\alpha_{i}+\gamma_{i}\right|-\frac{\pi}{2} \leq \frac{\pi}{2}$. If $-\frac{\pi}{2} \leq\left|\alpha_{i}+\gamma_{i}\right|-\frac{\pi}{2} \leq 0$, then it is concluded from (7) that $\frac{d}{d t}\left\|P-q_{i}(t)\right\| \leq 0$ and the proof is complete. If, on the other hand, $0<\left|\alpha_{i}+\gamma_{i}\right|-\frac{\pi}{2} \leq \frac{\pi}{2}$, then it follows from (7) and the inequality $\sin x<x$ for all $x \in\left(0, \frac{\pi}{2}\right]$, that

$$
\frac{d}{d t}\left\|P-q_{i}(t)\right\| \leq\left\|r_{i}\right\|\left(\left|\alpha_{i}+\gamma_{i}\right|-\frac{\pi}{2}\right) \cos \alpha_{i} \leq r_{M}\left|\alpha_{i}\right|
$$

which completes the proof.

Lemma 9: Define $\quad \alpha_{M}=\max _{i \in \mathbb{N}_{n}}\left|\alpha_{i}(0)\right| \quad$ and $T_{M}=\max \left\{\ln \frac{2 \alpha_{M}}{\pi}, 0\right\}$. Then, for any $t_{1} \geq T_{M}$ and any $t_{2}>t_{1}, R^{P}\left(t_{2}\right) \leq R^{P}\left(t_{1}\right)+r_{M} \alpha_{M} e^{-t_{1}}$.

Proof. Let $I$ denote the set of all agents at distance $R^{P}(t)$ from $P$ at time $t$ (i.e., the set of farthest agents from $P$ at time 
$t$ ). Then, it can be easily shown that

$$
\frac{d^{+}}{d t} R^{P}(t)=\max _{i \in I} \frac{d}{d t}\left\|P-q_{i}(t)\right\|
$$

To find an upper bound for $\frac{d^{+}}{d t} R^{P}(t)$, first note that $\alpha_{i}(t)=$ $\alpha_{i}(0) e^{-t}$ (since $\dot{\alpha}_{i}=-\alpha_{i}$ ), and hence $\left|\alpha_{i}(t)\right| \leq \frac{\pi}{2}$ for $t \geq$ $T_{M}$. Now, using Lemma 8 along with (8) yields $\frac{d^{+}}{d t} R^{P}(t) \leq$ $r_{M} \alpha_{M} e^{-t}$, for any $t \geq T_{M}$. By integrating both sides of this relation from $t_{1}$ to $t_{2}$, one can obtain $R^{P}\left(t_{2}\right)-R^{P}\left(t_{1}\right) \leq$ $r_{M} \alpha_{M}\left(e^{-t_{1}}-e^{-t_{2}}\right) \leq r_{M} \alpha_{M} e^{-t_{1}}$, which completes the proof.

The immediate result of the above lemma is that under the proposed control law the agents evolve in a bounded region in the plane. Note, however, that unlike the case of singleintegrator agents in [11], the convex hull of the agents in the case of unicycles is not necessarily contracting. This is due to the fact that when the heading of agent $i$ is not exactly in the same direction as $r_{i}$ (i.e., the angle $\alpha_{i}$ is nonzero), then the agent may not move toward the convex hull of its neighbors. Therefore, the method used in [11] (which is mainly based on the contracting property of the convex hull of agents) cannot be directly employed here to deduce the convergence of the agents to consensus. However, it is shown in the sequel that for the proposed controller, the dynamics of the positive limit set of the closed-loop system (see [12] for the definition of the positive limit set) reduces to that of the connectivity preserving controllers for single-integrator agents studied in [5]. Thus, the results of [11] can be used in this case to deduce convergence to consensus. To this end, the following lemma is borrowed from [12].

Lemma 10: If a solution $x(t)$ of $\dot{x}=f(x)$ belongs to a bounded domain $D$ for $t \geq 0$, then its positive limit set $L^{+}$ is nonempty, compact, and invariant. Also, $x(t)$ approaches $L^{+}$as $t \rightarrow \infty$.

The dynamics of the agents under the proposed control strategy can be written as

$$
\begin{aligned}
\dot{q}_{i} & =\frac{1}{2}\left(\operatorname{Rot}\left(2 \alpha_{i}\right)+I_{2}\right) r_{i} \\
\dot{\alpha}_{i} & =-\alpha_{i}
\end{aligned}
$$

Denote by $L^{+}$the positive limit set for a solution $\left[q^{T}(t) \alpha^{T}(t)\right]^{T}$ of (9), where $q(t)=\left[q_{1}^{T}(t) \ldots q_{n}^{T}(t)\right]^{T}$ and $\alpha(t)=\left[\alpha_{1}(t) \ldots \alpha_{n}(t)\right]^{T}$. Note that $L^{+}$possesses the properties described in Lemma 10 because the solution of (9) evolves in a bounded region (as shown earlier). For any $\left[p^{T} \beta^{T}\right]^{T} \in L^{+}$, there is a sequence $\left\{t_{n}\right\}$ with $t_{n} \rightarrow \infty$ as $n \rightarrow \infty$, such that $q\left(t_{n}\right) \rightarrow p$ and $\alpha\left(t_{n}\right) \rightarrow \beta$ as $n \rightarrow \infty$. This implies that $\beta=0$ because $\alpha\left(t_{n}\right)=e^{-t_{n}} \alpha(0) \rightarrow 0$ as $t_{n} \rightarrow \infty$. As a result, for the solutions starting in $L^{+}$(and hence staying in $L^{+}$as this set is invariant), (9) reduces to

$$
\dot{p}_{i}=r_{i}=-\frac{\partial h_{i}}{\partial p_{i}}
$$

This is the same connectivity preserving controller developed for single-integrator agents in [5]. Theorem 1 from [5] is used in the next lemma to characterize some important properties of $L^{+}$.

Lemma 11: For any $\left[p^{T} 0^{T}\right]^{T} \in L^{+}$and any $(i, j) \in E$, the inequality $\left\|p_{i}-p_{j}\right\|<d$ holds.
Proof. By definition, for any $\left[\begin{array}{ll}p^{T} & 0^{T}\end{array}\right]^{T} \in L^{+}$, there is a sequence $\left\{t_{n}\right\}$ with $t_{n} \rightarrow \infty$ as $n \rightarrow \infty$, such that $q\left(t_{n}\right) \rightarrow p$. Since $\left\|q_{i}\left(t_{n}\right)-q_{j}\left(t_{n}\right)\right\|<d$ (because of connectivity preservation), hence $\left\|p_{i}-p_{j}\right\| \leq d$. Now, choose an arbitrary $\tau>0$ and let $p^{\tau}(t)$ be a solution of (10) which passes through $p$ at time $\tau$ (i.e. $p^{\tau}(\tau)=p$ ). It follows from the invariance property of $L^{+}$that $\left[\left(p^{\tau}(t)\right)^{T} 0^{T}\right]^{T} \in L^{+}$for all $t \geq 0$. In particular, $\left[\left(p^{\tau}(0)\right)^{T} 0^{T}\right]^{T} \in L^{+}$implies that $\left\|p_{i}^{\tau}(0)-p_{j}^{\tau}(0)\right\| \leq d$. Let $G_{d}(0)$ be the union of the edges $(i, j) \in E(G)$ for which $\left\|p_{i}^{\tau}(0)-p_{j}^{\tau}(0)\right\|=d$. Let also $G_{M}=\left(V_{M}, E_{M}\right)$ be the maximal induced subgraph of $G_{d}(0)$ such that $d_{i}\left(G_{M}\right) \geq 2$ for every $i \in$ $V_{M}$. Since $G$ is a tree, $G_{d}(0)$ is acyclic and thus $G_{M}$ is empty. Therefore, Theorem 1 from [5] yields $\left\|p_{i}^{\tau}(t)-p_{j}^{\tau}(t)\right\|<d$ for all $(i, j) \in E$ and $t>0$. The proof follows now on noting that $\left\|p_{i}-p_{j}\right\|=\left\|p_{i}^{\tau}(\tau)-p_{j}^{\tau}(\tau)\right\|$.

Theorem 2: Consider a team of $n$ unicycle agents in a 2D plane with the dynamics of the form (1), and the control law (3). Consider also a set of analytic functions $h_{i}, i \in \mathbb{N}_{n}$, satisfying the conditions given by (2) and (6), which are used to obtain the control parameters in (3) as discussed in the previous section. Moreover, assume that the information flow graph is a static tree. Then, the agents converge to consensus while preserving connectivity.

Proof. The first step of the proof is to show that there exists a constant vector $\bar{p}$, for which $\left[\begin{array}{ll}\bar{p}^{T} & 0^{T}\end{array}\right]^{T} \in L^{+}$and $\bar{p}_{1}=\bar{p}_{2}=$ $\ldots=\bar{p}_{n}$. To this end, let $p(t)$ be a solution to (10) starting from a point $p(0)$, where $\left[p^{T}(0) 0^{T}\right]^{T} \in L^{+}$. Since $L^{+}$is invariant, hence $\left[p^{T}(t) 0^{T}\right]^{T} \in L^{+}$for all $t \geq 0$. On the other hand, (10) can be written as

$$
\dot{p}_{i}=-\sum_{j \in N_{i}(G)} \beta_{i j}\left(p_{i}-p_{j}\right)
$$

where $\beta_{i j}=\frac{\partial h_{i}}{\partial \sigma_{i}}-\frac{\partial h_{i}}{\partial \pi_{i}} \pi_{i j}$. Lemma 11 along with (6) yields $\beta_{i j}>0$ for all $(i, j) \in E$. The stability of the system governed by (11) has been extensively studied in the literature (e.g., see [11]). Using Proposition 2 in [11], it is straightforward to show the convergence of $p(t)$ to a point $\bar{p}$ for which $\bar{p}_{1}=\bar{p}_{2}=\ldots=$ $\bar{p}_{n}:=\mathfrak{p}$. Now, one can conclude that $\left[\begin{array}{ll}\bar{p}^{T} & 0^{T}\end{array}\right]^{T} \in L^{+}$because $L^{+}$is a closed set according to Lemma 10 .

To complete the proof, it suffices to show that for the solution $\left[q^{T}(t) \alpha^{T}(t)\right]^{T}$ of (9), $q(t)$ converges to $\bar{p}$, or equivalently $R^{\mathfrak{p}}(t)=\max _{i \in \mathbb{N}_{n}}\left\|\mathfrak{p}-q_{i}(t)\right\| \rightarrow 0$ as $t \rightarrow \infty$. Since $\left[\begin{array}{ll}\bar{p}^{T} & 0^{T}\end{array}\right]^{T} \in L^{+}$, there is a sequence $\left\{t_{n}\right\}$ with $t_{n} \rightarrow \infty$ as $n \rightarrow \infty$, such that $q\left(t_{n}\right) \rightarrow \bar{p}$ as $n \rightarrow \infty$, implying that $R^{\mathfrak{p}}\left(t_{n}\right) \rightarrow 0$ as $n \rightarrow \infty$. For any $\varepsilon>0$, choose a sufficiently large number $n$ such that $t_{n}>T_{M}, r_{M} \alpha_{M} e^{-t_{n}}<\frac{\varepsilon}{2}$, and $R^{\mathfrak{p}}\left(t_{n}\right)<\frac{\varepsilon}{2}$. Then, it results from Lemma 9 that for every $t>t_{n}$ the relation $R^{\mathfrak{p}}(t) \leq R^{\mathfrak{p}}\left(t_{n}\right)+r_{M} \alpha_{M} e^{-t_{n}}<\varepsilon$ holds, which completes the proof of convergence of $q_{i}$ 's to $\mathfrak{p}$.

\section{Simulation Results}

Example 1: To verify the effectiveness of the controller proposed in Section IV, consider 6 unicycle agents with dynamics of the form (1) moving in a 2D plane, with the information flow graph $G$ depicted in Fig. 2. Assume that $d=1$, and that the initial position and heading of each agent is as shown in Fig. 2. Suppose that agent $i$ is using a controller 


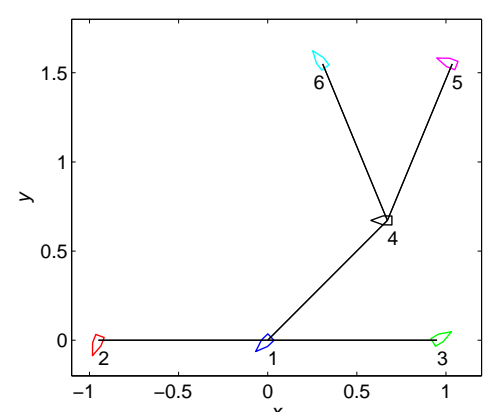

Fig. 2: The information flow graph $G$ along with the initial positions and headings of agents in Example 1.

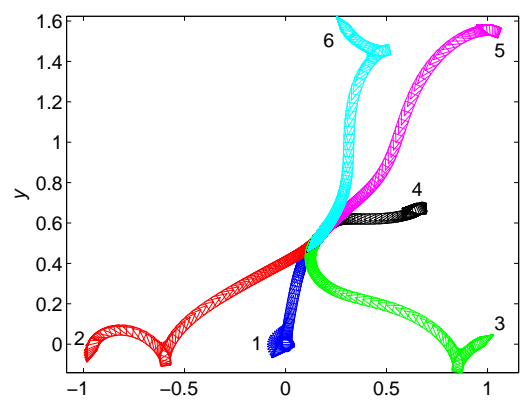

Fig. 3: The agents' planar motion in Example 1.

of the form (3), and that

$$
h_{i}\left(\sigma_{i}, \pi_{i}\right)=-\frac{\pi_{i}}{1+\sigma_{i}}, \quad i \in \mathbb{N}_{6}
$$

This function satisfies the conditions given in (2) and (6). Hence, the resultant controller is connectivity preserving and leads to consensus, according to Theorem 2.

The planar motion of the agents under the proposed controller is shown in Fig. 3. Denote the distance between agent $i$ and its neighbor $j$ by $d_{i j}$ (i.e., $d_{i j}:=\left\|q_{i}-q_{j}\right\|$ ). This distance is depicted in Fig. 4 for different agents as a function of time. While all initial distances are relatively close to $d$, the proposed controller keeps them less than $d$ for every $(i, j) \in E(G)$ at all times, as the agents converge to consensus. The translational and angular velocities of the agents are also depicted in Figs. 5 and 6, respectively.

\section{CONClusions}

This paper presents a connectivity preserving distributed control law for a network of unicycles. If two agents are initially located in the connectivity range, under the proposed control strategy they will remain connected at all times. The controller is designed in such a way that when an agent is

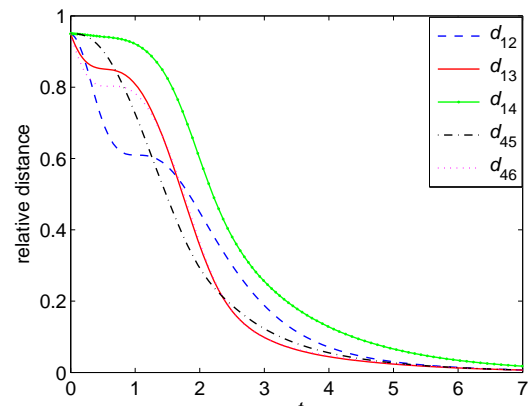

Fig. 4: The distances between the ${ }^{t}$ eighboring agents in Example 1.

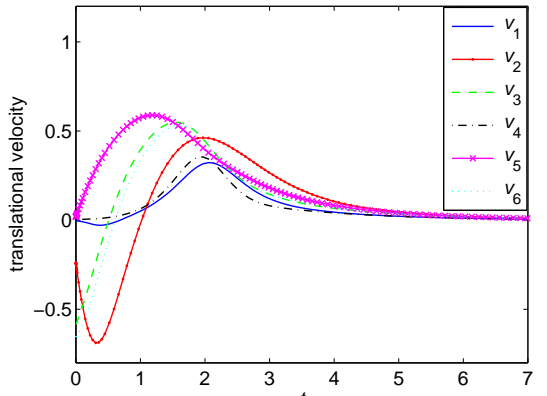

Fig. 5: The translational velocities of agents in Example 1.

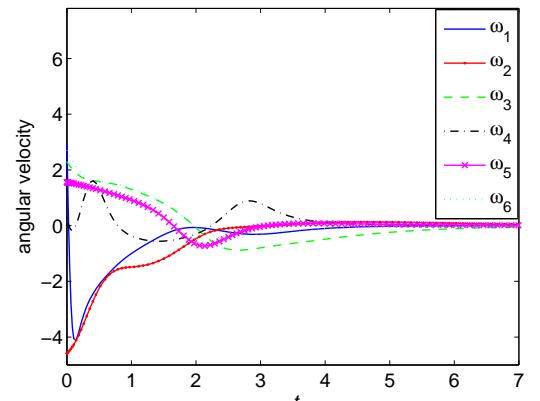

Fig. 6: The angular velocities of agents in Example 1.

about to lose connectivity with a neighbor, the lowest order derivative of the agent's position which is neither zero nor perpendicular to the corresponding edge, makes an acute angle with this edge, aiming to shrink it. Simulations confirm the validity of the theoretical results.

\section{REFERENCES}

[1] M. M. Zavlanos and G. J. Pappas, "Distributed connectivity control of mobile networks," IEEE Transactions on Robotics, vol. 24, no. 6, pp. 1416-1428, 2008.

[2] D. V. Dimarogonas and K. J. Kyriakopoulos, "Connectedness preserving distributed swarm aggregation for multiple kinematic robots," IEEE Transactions on Robotics, vol. 24, no. 5, pp. 1213-1223, 2008.

[3] M. Ji and M. Egerstedt, "Distributed coordination control of multiagent systems while preserving connectedness," IEEE Transactions on Robotics, vol. 23, no. 4, pp. 693-703, 2007.

[4] D. V. Dimarogonas and K. H. Johansson, "Bounded control of network connectivity in multi-agent systems," IET Control Theory and Applications, vol. 4, no. 8, pp. 1330-1338, 2010.

[5] A. Ajorlou, A. Momeni, and A. G. Aghdam, "A class of bounded distributed control strategies for connectivity preservation in multi-agent systems," IEEE Transactions on Automatic Control, vol. 55, no. 12, pp. 2828-2833, 2010.

[6] M. C. D. Gennaro and A. Jadbabaie, "Decentralized control of connectivity for multi-agent systems," in Proceedings of the 45th IEEE Conference on Decision and Control, 2006, pp. 3628-3633.

[7] P. Yang, R. A. Freeman, G. J. Gordon, K. M. Lynch, S. S. Srinivasa, and R. Sukthankar, "Decentralized estimation and control of graph connectivity for mobile sensor networks," Automatica, vol. 46, no. 2, pp. 390-396, 2010.

[8] M. M. Zavlanos, H. G. Tanner, A. Jadbabaie, and G. J. Pappas, "Hybrid control for connectivity preserving flocking," IEEE Transactions on Automatic Control, vol. 54, no. 12, pp. 2869-2875, 2009.

[9] M. M. Zavlanos, M. Egerstedt, and G. J. Pappas, "Graph theoretic connectivity control of mobile robot networks," Proceedings of the IEEE, vol. 99, no. 9, pp. 1525-1540, 2011.

[10] D. V. Dimarogonas and K. J. Kyriakopoulos, "On the rendezvous problem for multiple nonholonomic agents," IEEE Transactions on Automatic Control, vol. 52, no. 5, pp. 916-922, 2007.

[11] A. Ajorlou, A. Momeni, and A. G. Aghdam, "Sufficient conditions for the convergence of a class of nonlinear distributed consensus algorithms," Automatica, vol. 47, no. 3, pp. 625-629, 2011.

[12] H. K. Khalil, Nonlinear Systems, 3rd ed. New Jersey: Prentice Hall, 2002. 